\title{
Extended Serre Equations for Applications in Intermediate Water Depths
}

\author{
José Simão Antunes do Carmo*
}

IMAR, Institute of Marine Research, University of Coimbra, FCTUC, Department of Civil Engineering, Coimbra, Portugal

\begin{abstract}
The Serre or Green and Naghdi equations are fully-nonlinear and weakly dispersive and have a built-in assumption of irrotationality. However, like the standard Boussinesq equations, also Serre's equations are only valid for long waves in shallow waters. To allow applications in a greater range of $h_{0} / l$, where $h_{0}$ and $l$ represent, respectively, depth and wavelength characteristics, a new set of extended Serre equations, with additional terms of dispersive origin, is developed and tested in this work by comparisons with available experimental data. The equations are solved using an efficient finite-difference method, which consistency and stability are analyzed by comparison with a closed-form solitary wave solution of the Serre equations. All cases of waves propagating in intermediate water depths illustrate the good performance of the extended Serre equations with additional terms of dispersive origin. It is shown that the computed results are in conformity with the analytical ones and test data. An equivalent form of the Boussinesq type equations, also with improved linear dispersion characteristics, is solved using a numerical procedure similar to that used to solve the extended Serre equations.
\end{abstract}

Keywords: Serre equations, improved dispersion characteristics, intermediate water depths, finite differences, applications.

\section{INTRODUCTION}

The classical Boussinesq equations are valid for relatively low values of the frequency dispersion $\sigma=h_{0} / l$, where $h_{0}$ and $l$ represent, respectively, depth and wavelength characteristics. In fact, these equations are only strictly valid for shallow water conditions, so with $h_{0} / l$ not exceeding about 0.05 (or about 0.20 accepting phase velocity errors in the order of 5\%). Madsen et al. [1] and Madsen and Sørensen [2] included higher order terms of dispersive origin in the Boussinesq [3] equations, with adjustable coefficients for constant and variable depths, respectively, enabling applications in a wider range of values.

As an alternative to the Madsen and Sørensen [2] equations, Nwogu [4] obtained a new approximation, resulting from the vertical integration of the fundamental equations of the Fluid Mechanics, and then expanding in terms of the frequency dispersion $\sigma$ and nonlinearity $\varepsilon$ parameters, with $\varepsilon$ $=a / h_{0}$, being $a$ wave amplitude, and retaining terms until $O\left(\varepsilon, \sigma^{2}\right)$. To improve the dispersive characteristics of the new approach, [4] introduced a set of parameters which were calibrated by comparing the dispersion relation of the linearized model with the linear dispersion relation $\omega^{2}=g k \tanh (k h)$, allowing applications in the range $h_{0} / l \leq$ 0.48 , with an error for the phase velocity not greater than about $2 \%$. An efficient predictor-corrector finite-difference numerical scheme to solve Nwogu's equations, together with appropriate boundary conditions, is presented in [5].

\footnotetext{
*Address correspondence to this author at the IMAR, Institute of Marine Research, University of Coimbra, FCTUC, Department of Civil Engineering, Coimbra, Portugal; Tel: +351 239797 153; Fax: +351 239797 123;

E-mail: jsacarmo@dec.uc.pt
}

Wei et al. [6] and Gobbi et al. [7] derived fully nonlinear extensions of the Boussinesq equations, from which the Nwogu's approximation was recovered by neglecting higherorder terms. A numerical scheme similar to that of [5] is utilized, with the inclusion of extra nonlinear terms. Basically using the same high-order predictor-corrector scheme, [8] developed a numerical code (COULWAVE) based on Nwogu's equations for one and two layers. Parameterizations of bottom friction and wave breaking have been included in the code, as well as a moving boundary scheme to simulate wave runup and rundown.

Starting from the classical Boussinesq equations and adopting the methodology introduced by [1], Beji and Nadaoka [9] presented a new approach valid for applications until values of $h_{0} / l$ to the order of 0.25 , and still with acceptable errors in amplitude and phase velocity up to values of $h_{0} / l$ near 0.50 . A higher order of approximation, valid for values of $h_{0} / l$ to the order of 0.48 , is presented in [10]. Both approaches consider parameters that improve the linear dispersion characteristics of the standard Boussinesq equations. These parameters are derived by equating the dispersion relation of the linearized equations with the linear relation $\omega^{2}=g k \tanh (k h)$.

In this work, we follow the methodology introduced by [1], and adopted by [9], to extend the classical Serre or Green and Naghdi equations [11,12] for applications in intermediate water depths, up to frequency dispersion values $\sigma \approx 0.50$. An efficient numerical procedure is implemented to solve an extended approximation of the Boussinesq type and the new set of Serre equations for variable depth with improved dispersive characteristics.

Numerical results of a solitary wave with amplitudes $a / h_{0}=0.60$ and $a / h_{0}=0.30$ are compared, respectively, with 
a closed-form solitary wave solution of the Serre equations and with an approximate analytical solution of the classical Boussinesq equations. Results of a solitary wave travelling up a slope 1:50 and reflection on a vertical wall are compared and discussed with available experimental data. Then, the numerical results of an incident periodic wave in a domain with $h_{0} / l=0.50$, which is the limit between intermediate water depths and deep water conditions, and therefore already beyond the conditions of validity of Nwogu and Liu and Sun models, are presented and discussed.

Finally, numerical results of non-breaking periodic waves over an underwater bar are also presented and discussed through comparisons with test data available in the literature.

\section{MATHEMATICAL FORMULATION}

Boussinesq and Serre type equations are derived considering the fundamental equations of continuity and momentum in the vertical plane, relative to the flow of a nonviscous and non-compressible fluid subjected to the gravity action. In addition, the fundamental hypothesis of shallow water $(\sigma<<1)$, as well as, in the case of Boussinesq approximation, a relative elevation of the surface due to the waves $\left(\varepsilon=a / h_{0}\right)$ having a value close to the square of the relative depth $\left(\sigma=a / h_{0}\right)$, which means that $O(\varepsilon)=O\left(\sigma^{2}\right)$, is assumed. Under these hypotheses, considering suitable dimensionless variables, the fundamental equations are integrated in the vertical and expanded in terms of the small parameters $\sigma$ and $\varepsilon$, retaining terms up $O\left(\varepsilon, \sigma^{2}\right)$, thus the following dimensionless system is obtained (See [13] for details):

$$
\begin{aligned}
& \frac{\partial(\eta-\xi / \varepsilon)}{\partial t}+\frac{\partial[(1-\xi+\varepsilon \eta) \bar{u}]}{\partial x}=0 \\
& \frac{\partial \bar{u}}{\partial t}+\varepsilon \bar{u} \frac{\partial \bar{u}}{\partial x}+\frac{\partial \eta}{\partial x}+\sigma^{2} \frac{\partial \eta}{\partial x} \frac{\partial^{2} \xi}{\partial t^{2}}+\sigma^{2} \frac{\eta}{2} \frac{\partial^{3} \xi}{\partial x \partial t^{2}} \\
& \quad+\sigma^{2}\left[\frac{1-\xi}{2 \varepsilon} \frac{\partial^{3} \xi}{\partial x \partial t^{2}}-\frac{(1-\xi)^{2}}{3} \frac{\partial^{3} \bar{u}}{\partial x^{2} \partial t}+(1-\xi) \frac{\partial \xi}{\partial x} \frac{\partial^{2} \bar{u}}{\partial x \partial t}\right. \\
& \left.\quad+\frac{1-\xi}{2} \frac{\partial^{2} \xi}{\partial x^{2}} \frac{\partial \bar{u}}{\partial t}+(1-\xi) \frac{\partial^{2} \xi}{\partial x \partial t} \frac{\partial \bar{u}}{\partial x}+(1-\xi) \frac{\partial^{3} \xi}{\partial x^{2} \partial t} \bar{u}\right] \\
& \quad-\frac{\tau_{s}(\varepsilon \eta)-\tau_{b}(-1+\xi)}{\varepsilon \sigma(1-\xi+\varepsilon \eta)}=O\left(\sigma^{4}, \varepsilon^{2}\right)
\end{aligned}
$$

where $R$ is the Reynolds number, equal to $h_{0} c_{0} / v, c_{0}$ being the critical celerity, $c_{0}=\sqrt{g h_{0}}$. The system of equations (1) applies to the generation and propagation of waves in the most general terms, but only in shallow water conditions and small relative amplitudes, allowing the consideration of: $i$ ) bottom variations in time; ii) friction at bottom, and iii) stresses on the water surface. In dimensional variables, eliminating the bar over the $u$ variable to simplify writing and with a different notation, this approach is written as

$$
\begin{aligned}
& h_{t}+(h u)_{x}=0 \\
& u_{t}+u u_{x}+g \eta_{x}+\eta_{x} \xi_{t t}+0.5 \eta \xi_{x t t}+0.5\left(\zeta_{0}-\xi\right) \xi_{x t t} \\
& \quad-\left[\left(\zeta_{0}-\xi\right)^{2} / 3\right] u_{x x t}+\left(\zeta_{0}-\xi\right) \xi_{x} u_{x t}+0.5\left(\zeta_{0}-\xi\right) \xi_{x x} u_{t} \\
& \quad+\left(\zeta_{0}-\xi\right) \xi_{x t} u_{x}+\left(\zeta_{0}-\xi\right) \xi_{x x t} u-\left(\tau_{s}-\tau_{b}\right) /(\rho h)=0
\end{aligned}
$$

where $h(x, t)=\zeta_{0}-\xi(x)+\eta(x, t)$ is the flow depth, $\zeta_{0}$ is the free surface level at rest, $\xi(x)$ represents the bathymetry, $\eta(x, t)$ is the free surface elevation, $u(x, t)$ is the horizontal velocity, $t$ is time, $g$ is the gravitational acceleration, $\rho$ is the fluid density, $v$ is the kinematic viscosity, $\tau_{s}$ and $\tau_{b}$ represent friction stresses on free surface and at bottom, respectively. Considering the flow of a viscous fluid over a fixed bottom and negligible surface stresses, the system of equations (2) reduces to

$$
\begin{aligned}
& h_{t}+(h u)_{x}=0 \\
& u_{t}+u u_{x}+g(h+\xi)_{x}-\left[\left(\zeta_{0}-\xi\right)^{2} / 3\right] u_{x x t}+\left(\zeta_{0}-\xi\right) \xi_{x} u_{x t} \\
& \quad+0.5\left(\zeta_{0}-\xi\right) \xi_{x x} u_{t}+\tau_{b} /(\rho h)=0
\end{aligned}
$$

By an addition and subtraction process, [9] consider a dispersion parameter $\beta$ in the second equation of system (3), with a value obtained by comparison of the dispersion relation of the linearized form of this system with a second-order Padé expansion of the linear dispersion relation $\omega^{2} / g k=\tanh (k h)$. Then, using the approximation $u_{t}=-g \eta_{x}$ an improved set of Boussinesq equations for variable depth is obtained.

Using identical methodology, Liu and Sun [10] consider two dispersion parameters $\alpha$ and $\gamma$, which values are determined as explained below. Rearranging terms, and given that $\xi_{x}=-\left(\zeta_{0}-\xi\right)_{x}$, as well as $\xi_{x x}=-\left(\zeta_{0}-\xi_{x x}\right.$, the following approximation with improved dispersive characteristics is obtained

$$
\begin{aligned}
& h_{t}+(h u)_{x}=0 \\
& u_{t}+u u_{x}+g(h+\xi)_{x}-[(1+\alpha) / 2-(1+\gamma) / 6]\left[\left(\zeta_{0}-\xi\right)^{2}\right] u_{x x t} \\
& \quad-(1+\alpha)\left(\zeta_{0}-\xi\right)\left(\zeta_{0}-\xi\right)_{x} u_{x t}-0.5(1+\alpha)\left(\zeta_{0}-\xi\right)\left(\zeta_{0}-\xi\right)_{x x} u_{t} \\
& \quad-(\alpha / 2-\gamma / 6) g\left[\left(\zeta_{0}-\xi\right)^{2}\right] \eta_{x x x}-\alpha g\left(\zeta_{0}-\xi\right)\left(\zeta_{0}-\xi\right)_{x} \eta_{x x} \\
& \quad-0.5 \alpha g\left(\zeta_{0}-\xi\right)\left(\zeta_{0}-\xi\right)_{x x} \eta_{x}+\tau_{b} /(\rho h)=0
\end{aligned}
$$

After linearization of the equations system (4), [10] obtained the following dispersion relation

$$
\frac{\omega^{2}}{g k}=\frac{k h\left[1+(\alpha / 2-\gamma / 6) k^{2} h^{2}\right]}{1+(1+\alpha) k^{2} h^{2} / 2-(1+\gamma) k^{2} h^{2} / 6}
$$

Comparing equation (5) with the second-order Padé expansion of the linear Stokes dispersion relation $\omega^{2} / g k=\tanh (k h)$ $=k h\left(1+k^{2} h^{2} / 15\right) /\left[1+2(k h)^{2} / 5\right]$, the expression $\alpha / 2-\gamma / 6=1 / 15$ can be obtained. With $\beta=1 / 5$, as determined by [9], it follows that $\beta=(3 / 2) \alpha-(1 / 2) \gamma$. By a further comparison between the linear shoaling characteristic of the extended Boussinesq equations and that of the linear Stokes waves, the two parameters $\alpha$ and $\gamma$ have been optimized by [10] to be: $\alpha=0.1308$ and $\gamma=-0.0076$, and therefore $\beta=0.20$.

Under the assumptions made to obtain the system of equations (3), except the relative elevation of the free surface due to waves $\left(\varepsilon=a / h_{0}\right)$, which is now $O(\varepsilon)=1$ instead of $O(\varepsilon)<<1$, the equations of continuity and momentum (6) are 
obtained, in dimensional variables (known as Serre or Green and Naghdi equations for variable depth). See [14] for details.

$$
\begin{aligned}
& h_{t}+(u h)_{x}=0 \\
& u_{t}+u u_{x}+g \eta_{x}+\Omega u_{t}-h h_{x} u_{x t}-\frac{h^{2}}{3}\left(u_{x x t}-u_{x} u_{x x}+u u_{x x x}\right) \\
& \quad-h h_{x} u u_{x x}+\left[h\left(u_{x}\right)^{2}+\xi_{x x} u^{2}\right] \eta_{x}+\left(\Omega+h \xi_{x x}\right) u u_{x} \\
& +\frac{h}{2} \xi_{x x x} u^{2}+\tau_{b} /(\rho h)=0
\end{aligned}
$$

where $\Omega=h_{x} \xi_{x}+0.5 h \xi_{x x}+(\xi)^{2}, h=\zeta_{0}-\xi+\eta$ and, as stated before, $\zeta_{0}$ is water surface level at rest, $\xi$ represents bathymetry, $\eta$ is wave surface elevation, $u$ is depth-averaged velocity, and $g$ is gravitational acceleration.

The procedure used to obtain the extended Boussinesq approximation (4) is now utilized to improve the dispersion characteristics of the model (6). Adding and subtracting terms of dispersive origin, with time derivatives of $u$ in the second equation of (6), considering the parameters $\alpha$ and $\beta$, with $\beta=1.5 \alpha-0.5 \gamma$, as shown above, and replacing $u_{t}$ with $-\mathrm{g} \eta_{x}$, allows us to obtain the new system of Serre equations (7) with improved linear dispersion characteristics

$$
\begin{aligned}
& h_{t}+(u h)_{x}=0 \\
& u_{t}+u u_{x}+g(h+\xi)_{x}+(1+\alpha)\left(\Omega u_{t}-h h_{x} u_{x t}\right)-(1+\beta) \frac{h^{2}}{3} u_{x x t} \\
& \quad+\alpha g \Omega(h+\xi)_{x}-\alpha g h h_{x}(h+\xi)_{x x}-\beta g \frac{h^{2}}{3}(h+\xi)_{x x x}-h h_{x} u u_{x x} \\
& \quad+\frac{h^{2}}{3}\left(u_{x} u_{x x}-u u_{x x x}\right)+h\left(u_{x}\right)^{2}(h+\xi)_{x}+\xi_{x x} u^{2}(h+\xi)_{x} \\
& \quad+\left(\Omega+h \xi_{x x}\right) u u_{x}+\frac{h}{2} \xi_{x x x} u^{2}+\tau_{b} /(\rho h)=0
\end{aligned}
$$

As above, $\alpha=0.1308$ and $\beta=0.20$.

\section{NUMERICAL FORMULATION}

In order to solve the systems of equations (4) and (7), the terms containing derivatives in time of the $u$ variable are grouped. Those systems of equations are re-written according to the following equivalent forms

a) From system (4) - extended Boussinesq approximation

(a) $h_{t}+(u h)_{x}=0$

(b)

$$
\begin{aligned}
r_{t}= & -u u_{x}-g(h+\xi)_{x}+(\alpha / 2-\gamma / 6) g\left(\zeta_{0}-\xi\right)^{2} \eta_{x x x} \\
& +\alpha g\left(\zeta_{0}-\xi\right)\left(\zeta_{0}-\xi\right)_{x} \eta_{x x}+0.5 \alpha g\left(\zeta_{0}-\xi\right)\left(\zeta_{0}-\xi\right)_{x x} \eta_{x} \\
& -\tau_{b} /(\rho h)
\end{aligned}
$$

(c)

$$
\begin{gathered}
u-(1 / 3+\alpha / 2-\gamma / 6)\left(\zeta_{0}-\xi\right)^{2} u_{x x}-(1+\alpha)\left(\zeta_{0}-\xi\right)\left(\zeta_{0}-\xi\right)_{x} u_{x} \\
-0.5(1+\alpha)\left(\zeta_{0}-\zeta\right)\left(\zeta_{0}-\xi\right)_{x x} u=r
\end{gathered}
$$

b) From system (7) - extended Serre model

(a) $h_{t}+(u h)_{x}=0$

$$
\begin{aligned}
q_{t}+ & \left\{u q-\frac{1}{2}\left[u^{2}+(1+2 a) h^{2}\left(u_{x}\right)^{2}\right.\right. \\
& \left.\left.+(1+2 \alpha)\left(\xi_{x}\right)^{2} u^{2}-h \xi_{x}\left(u^{2}\right)_{x}\right]\right\}_{x} \\
& +\left[g(1+a \Omega)+\alpha h u u_{x x}\right] \eta_{x}-\alpha g h h_{x} \eta_{x x}-\beta g \frac{h^{2}}{3} \eta_{x x x} \\
& -\frac{a}{2}\left[\left(h \xi_{x x} u^{2}\right)_{x}+h_{x} \xi_{x x} u^{2}-h \xi_{x x} u u_{x}\right] \\
& +\left(\alpha-\frac{\beta}{3}\right) h^{2} u_{x} u_{x x}+\beta \frac{h^{2}}{3} u u_{x x x}+\tau_{b} /(\rho h)=0
\end{aligned}
$$

(c) $[1+(1+\alpha) \Omega] u-(1+\alpha) h h_{x} u_{x}-(1+\beta) \frac{h^{2}}{3} u_{x x}=q$

(d) $\Omega=\xi_{x} \eta_{x}+\frac{1}{2} h \xi_{x x}$

where $\eta=\xi+h-\zeta_{0}$, and therefore all space derivatives of $\eta$ are easily obtained.

To compute the solution of equation system (4) (values of the variables $h$ and $u$ at time $t+\Delta t$ ) we use a numerical procedure based on the following scheme, itself based on the last system of equations (8), for variables $h, r$ and $u$ [15]:

1- The first equation (8a) allows us to predict the values of variable $h\left(h_{p}^{t+\Delta t}\right)$ and, therefore, $\eta_{p}^{t+\Delta t}$ using a semiimplicit scheme, considering the known values of $h^{t}$ and $u^{t}$ in the whole domain.

2- The second equation (8b) allows the explicit prediction of the variable values $r\left(r_{p}^{t+\Delta t}\right)$, considering the values of $\tilde{h}^{t+\theta \Delta t}=(1-\theta) h^{t}+\theta h_{p}^{t+\Delta t}, \quad \tilde{\eta}^{t+\theta \Delta t}=(1-\theta) \eta^{t}+\theta \eta_{p}^{t+\Delta t}, \quad u^{t}$ and $r^{t}$ known in the whole domain.

3- The third equation (8c) allows us to obtain the values of variable $u$ at time $t+\Delta t\left(u^{t+\Delta t}\right)$ using a semi-implicit scheme, considering the predicted values of $r\left(r_{p}^{t+\Delta t}\right)$ in the whole domain.

4- The first equation (8a) allows us to compute the depth $h$ at time $t+\Delta t\left(h^{t+\Delta t}\right)$, taking into account the values of variable $h^{t}$ and the mean values $\bar{u}^{t+\theta \Delta t}=(1-\theta) u^{t}+\theta u^{t+\Delta t}$ known in the whole domain.

5- Finally, the second equation (8b) allows us to compute values of variable $r$ at time $t+\Delta t\left(r^{t+\Delta t}\right)$, taking into account the values of variable $r^{t}$ and the mean values $\bar{h}^{t+\theta \Delta t}=(1-\theta) h^{t}+\theta h^{t+\Delta t}$ and $\bar{u}^{t+\theta \Delta t}=(1-\theta) u^{t}+\theta u^{t+\Delta t}$ known in the whole domain.

Schematically, in a finite-difference form, the equivalent system of equations (8), for variables $h, r$ and $u$, may be written as follows [see $[13,16]$ for details]:

$$
\begin{aligned}
-\frac{u_{i}}{4 \Delta x} h_{i-1}^{n+1}+\left[\frac{1}{\Delta t}+\frac{1}{2}\left(\frac{\partial u}{\partial x}\right)_{i}\right] & h_{i}^{n+1}+\frac{u_{i}}{4 \Delta x} h_{i+1}^{n+1} \\
& =\left[\frac{1}{\Delta t}-\frac{1}{2}\left(\frac{\partial u}{\partial x}\right)_{i}\right] h_{i}^{n}-\frac{u_{i}}{2}\left(\frac{\partial h}{\partial x}\right)_{i}^{n}
\end{aligned}
$$




$$
\begin{aligned}
h_{i}^{n+\theta} & =(1-\theta) h_{i}^{n}+\theta h_{i}^{n+1} \\
\eta_{i}^{n+\theta} & =(1-\theta) \eta_{i}^{n}+\theta \eta_{i}^{n+1} \\
r_{i}^{n+1}= & r_{i}^{n}-\left[u_{i}\left(\frac{\partial u}{\partial x}\right)_{i}+g\left(\frac{\partial h}{\partial x}+\frac{\partial \xi}{\partial x}\right)_{i}\right. \\
& -\left(\frac{\alpha}{2}-\frac{\gamma}{6}\right) g\left(\zeta_{0}-\xi\right)^{2}\left(\frac{\partial^{3} \eta}{\partial x^{3}}\right)_{i}-\alpha g\left(\zeta_{0}-\xi\right)\left(\zeta_{0}-\xi\right)_{x}\left(\frac{\partial^{2} \eta}{\partial x^{2}}\right)_{i}(10) \\
& \left.\left.-\alpha g \frac{\left(\zeta_{0}-\xi\right)}{2}\left(\zeta_{0}-\xi\right)_{x x}\left(\frac{\partial \eta}{\partial x}\right)_{i}+\left(\frac{\tau_{b}}{\rho h}\right)\right]_{i}\right] \Delta t \\
{\left[-\left(1+\frac{3 \alpha}{2}-\frac{\gamma}{2}\right) \frac{\left(\zeta_{0}-\xi\right)^{2}}{3(\Delta x)^{2}}+\left.(1+\alpha) \frac{\left(\zeta_{0}-\xi\right)}{2 \Delta x} \frac{\partial\left(\zeta_{0}-\xi\right)}{\partial x}\right|_{i}\right]_{i-1}^{n+1} } & +\left[1+2\left(1+\frac{3 \alpha}{2}-\frac{\gamma}{2}\right) \frac{\left(\zeta_{0}-\xi\right)^{2}}{3(\Delta x)^{2}}-\left.(1+\alpha) \frac{\left(\zeta_{0}-\xi\right)}{2} \frac{\partial^{2}\left(\zeta_{0}-\xi\right)}{\partial x^{2}}\right|_{i}\right]_{i}^{n+1} \\
& -\left[\left(1+\frac{3 \alpha}{2}-\frac{\gamma}{2}\right) \frac{\left(\zeta_{0}-\xi\right)^{2}}{3(\Delta x)^{2}}+\left.(1+\alpha) \frac{\left(\zeta_{0}-\xi\right)}{2 \Delta x} \frac{\partial\left(\zeta_{0}-\xi\right)}{\partial x}\right|_{i}\right]_{i+1}^{n+1}=r_{i}^{n+1} \\
u_{i}^{n+\theta} & =(1-\theta) u_{i}^{n}+\theta u_{i}^{n+1}
\end{aligned}
$$

The third equation of (10) is explicit in relation to the $r$ variable, and the resulting systems of equations for the first and third equations ( $h$ and $u$ variables) are of three-diagonal form. At each interior point $i$ the first, second and third-order spatial derivatives are approximated through centred differences and the time derivatives are approximated using forward differences. Considering a uniform grid size $\Delta x$, such that $x_{i}=i \Delta x, i=1, \ldots, N$, and a generic variable $\mho$, the spatial difference operators are defined

$$
\begin{aligned}
& \left.v_{x}\right|_{i}=\frac{v_{i+1}-v_{i-1}}{2 \Delta x} \\
& \left.v_{x x}\right|_{i}=\frac{v_{i+1}-2 v_{i}+v_{i-1}}{(\Delta x)^{2}} \\
& \left.v_{x x x}\right|_{i}=\frac{v_{1+2}-2 v_{1+1}+2 v_{i-1}-v_{i-2}}{2(\Delta x)^{3}}
\end{aligned}
$$

In order to solve the set of equations (9), we assume that all values of $h_{i}$ and $u_{i}, i=1, N$, are known in the whole domain at time $n \Delta t$. Knowing all values of these variables, the equations (9c) and (9d) are used to obtain the first values of $\Omega_{i}$ and $q_{i}$ in the whole domain. Then, we use a numerical procedure based on the following steps, in which the index $p$ means predicted values:

1- The first equation (9a) is used to predict the variable values $h_{p i}$ at time $t+\Delta t\left(h_{p i}^{t+\Delta t}\right)$ and, therefore, $\eta_{p}^{t+\Delta t}$ in the whole domain.

2- The second equation (9b) makes it possible to predict the variable values $q_{p i}$ at time $t+\Delta t\left(q_{p i}^{t+\Delta t}\right)$, taking into account the values $\tilde{h}_{i}^{t+\Delta t}=0.5\left(h_{i}^{t}+h_{p i}^{t+\Delta t}\right) \quad$ and $\tilde{\eta}_{i}^{t+\Delta t}=0.5\left(\eta_{i}^{t}+\eta_{p i}^{t+\Delta t}\right)$, namely for $\Omega_{i}$ in the whole domain.
3- The third equation (9c) makes it possible to compute the mean-averaged velocity values $u_{i}^{t+\Delta t}$ at time $t+\Delta t$, taking into account the predicted values $h_{p i}^{t+\Delta t}$ and $q_{p i}^{t+\Delta t}$, namely for $\Omega_{i}$ in the whole domain.

4- The first operation (step 1) is repeated in order to improve the accuracy of the variable values $h_{i}$ at time $t+\Delta t$ $\left(h_{p t}^{t+\Delta t}\right)$, using the mean values $\bar{u}_{i}^{t+\Delta t}=0.5\left(u_{i}^{t}+u_{i}^{t+\Delta t}\right)$ in the whole domain.

5- Finally, the second operation (step 2) is repeated in order to improve the accuracy of the variable values $q_{i}$ at time $t+\Delta t\left(q_{i}^{t+\Delta t}\right)$, taking into account the mean values $\bar{h}_{i}^{t+\Delta t}=0.5\left(h_{i}^{t}+h_{i}^{t+\Delta t}\right), \quad \bar{\eta}_{i}^{t+\Delta t}=0.5\left(\eta_{i}^{t}+\eta_{i}^{t+\Delta t}\right) \quad$ and $\bar{u}_{i}^{t+\Delta t}=0.5\left(u_{i}^{t}+u_{i}^{t+\Delta t}\right)$ in the whole domain.

The convective terms $(u h)_{x}$ and $(u q)_{x}$ in equations (9a) and $9 \mathrm{~b}$ ) are approximated through centred schemes in space and time for variables $h$ and $q$. These terms are written in the following form:

$$
\begin{aligned}
& (u h)_{x}=u_{i}^{t}\left(\frac{h_{i+1}^{t}-h_{i-1}^{t}+h_{i+1}^{t+\Delta t}-h_{i-1}^{t+\Delta t}}{4 \Delta x}\right)+\frac{1}{2}\left(h_{i}^{t}+h_{i}^{t+\Delta t}\right)\left(\frac{u_{i+1}^{t}-u_{i-1}^{t}}{2 \Delta x}\right) \\
& (u q)_{x}=u_{i}^{t}\left(\frac{q_{i+1}^{t}-q_{i-1}^{t}+q_{i+1}^{t+\Delta t}-q_{i-1}^{t+\Delta t}}{4 \Delta x}\right)+\frac{1}{2}\left(q_{i}^{t}+q_{i}^{t+\Delta t}\right)\left(\frac{u_{i+1}^{t}-u_{i-1}^{t}}{2 \Delta x}\right)
\end{aligned}
$$

All finite-difference equations are implicit. Therefore, the solution of system (9) requires, in each time step, the computation of five three-diagonal systems of N-2 equations (steps 1 to 5), which are easily computed using the three-diagonal matrix algorithm (TDMA), also known as the Thomas algorithm. The stability condition to be observed can be expressed in terms of number of Courant, and is given by

$$
C_{R}=\sqrt{g h} \frac{\Delta t}{\Delta x}<1.0
$$

The best results are obtained by satisfying the condition $C_{R}<1.0$, with $\Delta x \leq l / 40$.

For waves of small relative amplitude, the input boundary condition for the velocity can be obtained from the elevation of the free surface and using the continuity equation; the resulting equation is

$$
u(0, t)=\frac{\omega}{k h_{0}\left[1+(\alpha / 2-\gamma / 6)\left(k h_{0}\right)^{2}\right]} \eta(0, t)
$$

where $k=2 \pi / l$ is the wave number.

In intermediate water depths $\left(0.05 \leq h_{0} / l \leq 0.50\right)$, and also for waves of small relative amplitude, a relation between $u(0, t)$ and $\eta(0, t)$ can be expressed as

$$
u(0, t)=\frac{g T}{2 \pi} \tanh \left(\frac{2 \pi h_{0}}{l}\right) \frac{1}{h_{0}} \eta(0, t)
$$

Both approximations (14) and (15) are acceptable. However, approximation (14) should be preferred, since it is ac- 


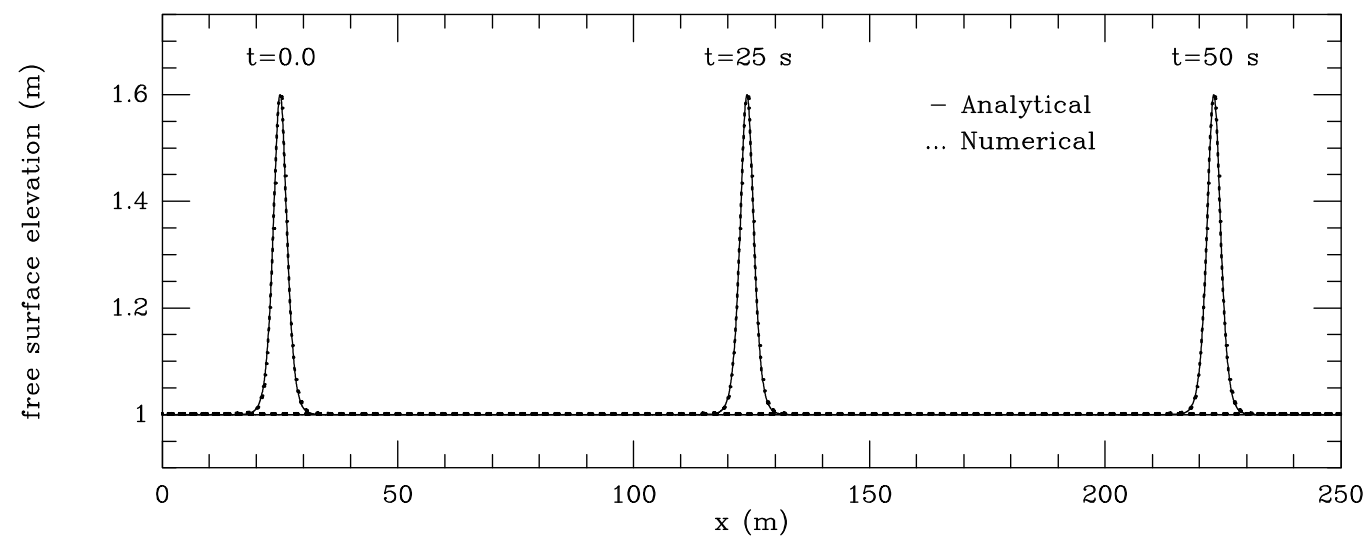

Fig. (1). Solitary wave computation for a wave with $a / h_{0}=0.60$. Comparison of the analytic solution (19) $(-)$ with numerical results of model (7) with $\alpha=\beta=0(\cdots \cdots)$.

cording to the equations used. Both of them allow mono- and bi-chromatic wave inputs. Different kinds of waves may be used. Input signals like $\eta(0, t) / \eta_{\max }=\cos (2 \pi \Delta f t)(2 \pi \Delta f t)$ $\cos (2 \pi \Delta f)$ (bi-chromatic wave group of $\Delta)$ or $\eta(0, t) / \eta_{\max }=(1 / n)\left\{\cos \left(2 \pi f_{1} t\right)+\cos \left(2 \pi f_{2} t\right)\right\} \quad$ (wavetrains consisting of consecutive small and large waves, if $f_{1} \neq f_{2}$ ) allow us to simulate different wave patterns. In these equations $f$ is the frequency and $\eta_{\max }$ is the maximum displacement of a random wave generator. For mono-chromatic waves, the water-surface elevation reduces to $\eta(0, t) / \eta_{\max }=\cos (2 \pi f t)$.

In order to permit the outgoing waves to be radiated offshore, the following relationship is applied:

$f_{n 1}-C \eta=-2 C \eta_{I}$

$u\left(h_{0}+\eta\right)=f_{n 1}$

where $f_{n 1}=$ outgoing normal flux, $\eta=$ actual sea surface elevation, $\eta_{I}=$ elevation of the incident wave, and $C=$ wave celerity.

At the output boundary, in order for the reflected wave amplitude to be identical to zero, a linearized non-dispersive form of the $\mathrm{KdV} \eta_{t}+c_{0} \eta_{x}=0$ [17], where $c_{0}=\omega / k$, has to be satisfied.

\section{APPLICATIONS}

\section{Comparisons with Analytical Solutions}

In order to test the above numerical procedures (8) and (9), used to solve the systems of equations (4) and (7), some comparisons with known analytical solutions have been made. For this purpose, the numerical procedure used to solve the classical Boussinesq equations is validated through an approximate solitary wave solution of the form

$$
\eta(x, t)=a \operatorname{sech}^{2}\left\{\sqrt{\frac{3 a}{4 h_{0}^{3}}}\left[x-\sqrt{g\left(h_{0}+a\right)} t-x_{0}\right]\right\}
$$

and to validate system (7), using procedure (9) with $\alpha=\beta=0$, a closed-form solitary wave solution of the Serre equations is used, which is expressed as

$h(x, t)=h_{0}+a \operatorname{sech}^{2}\left[K\left(x-C t-x_{0}\right)\right]$
$u=C\left(1-h_{0} / h\right)$

where $h_{0}=\zeta_{0}-\xi$ is the water depth at rest, $x_{0}$ is the initial position of the crest, $a$ is the wave amplitude, $K=\sqrt{3 a /\left[4 h_{0}^{2}\left(h_{0}+a\right)\right]}, \quad C=c_{0} \sqrt{\left(1+a / h_{0}\right)} \quad$ and $c_{0}=\sqrt{g h_{0}}$.

The numerical models that use the procedures (8) and (9) to solve the systems of equations (4) and (7) have been applied to propagate a solitary wave in a channel $1.0 \mathrm{~m}$ depth and $250 \mathrm{~m}$ long, with the initial position of the crest wave $x_{0}$ initially located at $25 \mathrm{~m}$ from the origin. Fig. (1) shows a comparison between numerical results, using procedure (9), and the Serre solution (19) for a wave with

$$
\mathrm{a} / h_{0}=0.60
$$

$a / h_{0}=0.60$, while Fig. (2) shows a comparison of the numerical results obtained using procedure (8) and the Boussinesq approximate solution (18) for the ratio $a / h_{0}=0.30$. Both computations were carried out with $\Delta t=0.010 \mathrm{~s}$. As shown below, the agreement between the numerical results of system (7), using procedure (9) with $\alpha=\beta=0$, with the analytical solution (19), for the ratio $a / h_{0}=0.60$, is perfect as much in wave amplitude as in phase, Fig. (1).

Although there is a good agreement for the wave heights, a slight loss in phase accuracy is most pronounced in Fig. (2), for the Boussinesq approximation, as shown for $t=60 \mathrm{~s}$ in a zoom, Fig. (3).

A parameter suitable to show how the computed values $\left(V_{c i}\right)$ differ from the analytical solution values $\left(V_{a i}\right)$ is the root mean square error (RMSE), given by 


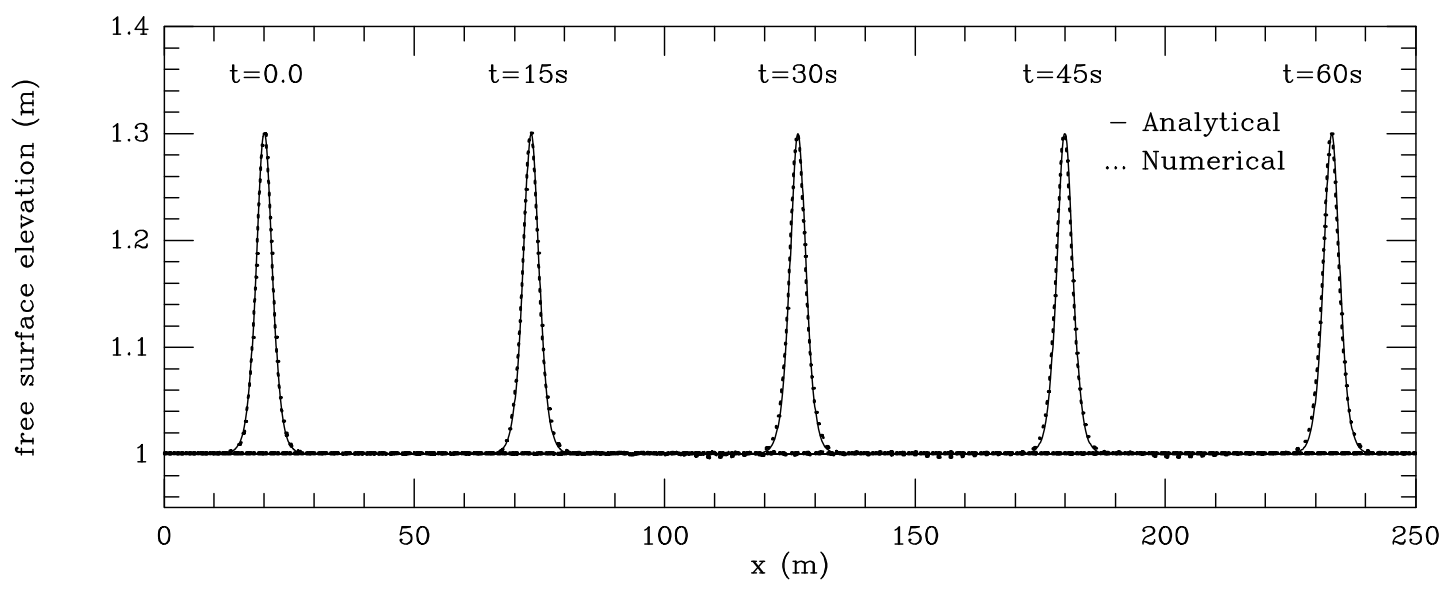

Fig. (2). Solitary wave computation for a wave with $a / h_{0}=0.30$. Comparison of the approximate analytic solution (18) ( $\longrightarrow$ ) with numerical results of model (4) with $\alpha=\gamma=0(\cdots \cdots)$.

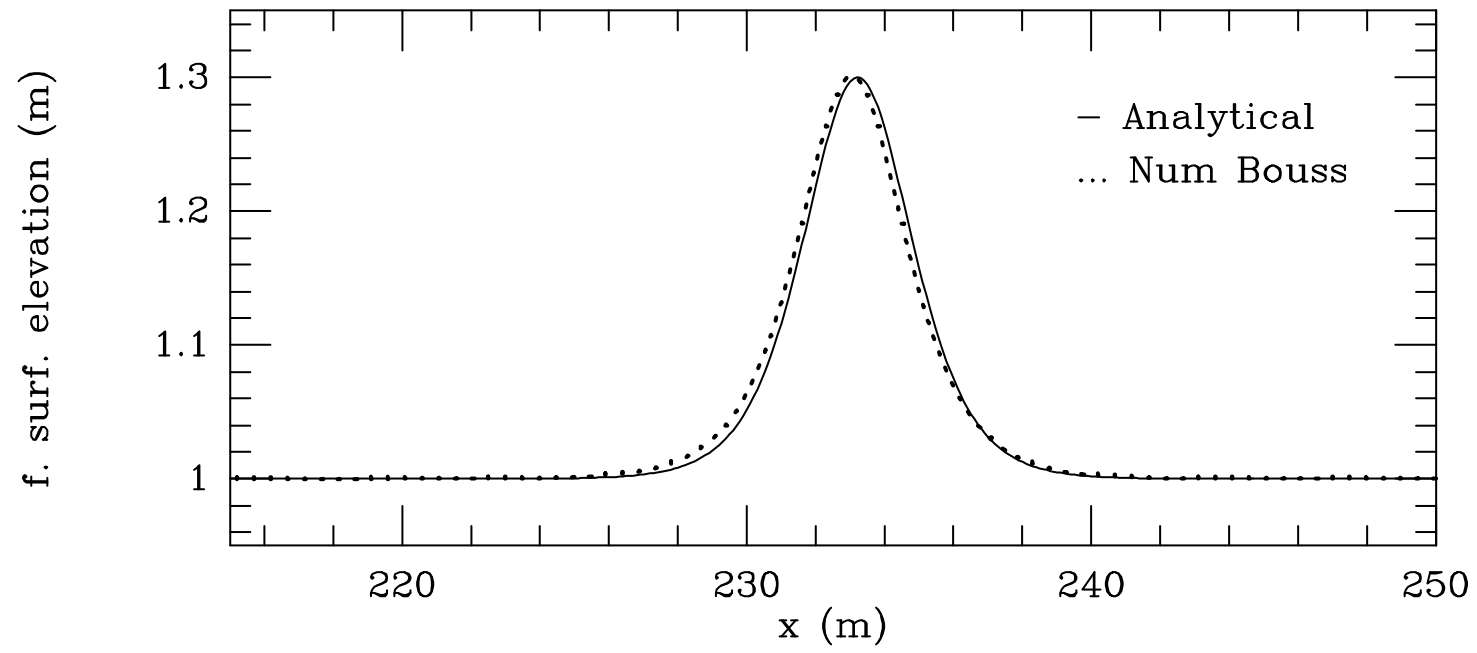

Fig. (3). Solitary wave computation for a wave with $a / h_{0}=0.30$, at time $t=60.0 \mathrm{~s}$. Comparison of the approximate analytic solution (18) ( - ) with numerical results of model (4) with $\alpha=\gamma=0(\cdots \cdots)$.

$R M S E=\sqrt{\frac{1}{N} \sum_{i=1}^{N}\left(V_{c i}-V_{a i}\right)^{2}}$

This parameter ranges from 0 to infinity, taking the value 0 for a perfect fit. RMSE values equal to $0.0007 \mathrm{~m}$ and $0.0027 \mathrm{~m}$ were found for the wave height in cases of Fig. (1), at time $\mathrm{t}=50 \mathrm{~s}$ (Serre solution for the ratio $a / h_{0}=0.60$ ), and Fig. (2), at time $\mathrm{t}=60 \mathrm{~s}$ (Boussinesq solution for the ratio $\left.a / h_{0}=0.30\right)$, respectively. Regarding the phase, there is a loss of approximately $0.025 \mathrm{~s}$ in Fig. (1) and of $0.20 \mathrm{~s}$ in Fig. (2).

\section{Incident Wave in Quasi-Deep Water Conditions}

As shown in [10], for the system (4), this model and the system of equations (7) are valid in intermediate waters and up to quasi-deep water conditions $\left(h_{0} / l \leq 0.48\right)$. Fig. (4) shows results of models (8) and (9) in a depth gauge for a periodic wave of amplitude $a=0.025 \mathrm{~m}$, period $T=0.85 \mathrm{~s}$ and wavelength $l=1.12 \mathrm{~m}$, propagating into an initially undisturbed region of constant depth $h_{0}=0.56 \mathrm{~m}$, thus in quasi-deep water conditions. The gauge was located at $x=5.0 \mathrm{~m}$ from the inlet section of a channel. The computational domain was discretized with a uniform grid interval $\Delta x=0.05 \mathrm{~m}$ and a time step $\Delta t=0.010 \mathrm{~s}$ was used.

As shown in Fig. (4), the results obtained with both approaches are very close. A steady periodic flow has been established and the linearized non-dispersive form of the $\mathrm{KdV} \eta_{t}+c_{0} \eta_{x}=0$ proved to be suitable for output boundary. There is only a slight improvement on stability of the wave using the extended Serre model with improved dispersion characteristics.

\section{Solitary Wave Travelling up a Slope and Reflection on a Vertical Wall}

Experimental data and numerical results are available for a solitary wave propagating on the bathymetry shown in Fig. (5) $[18,19]$. It shows a constant depth before $x=55 \mathrm{~m}$ and a slope 1:50 between $x=55 \mathrm{~m}$ and $x=75 \mathrm{~m}$. An impermeable vertical wall is placed at $x=75 \mathrm{~m}$, corresponding to fully reflecting boundary conditions. A solitary wave of amplitude 


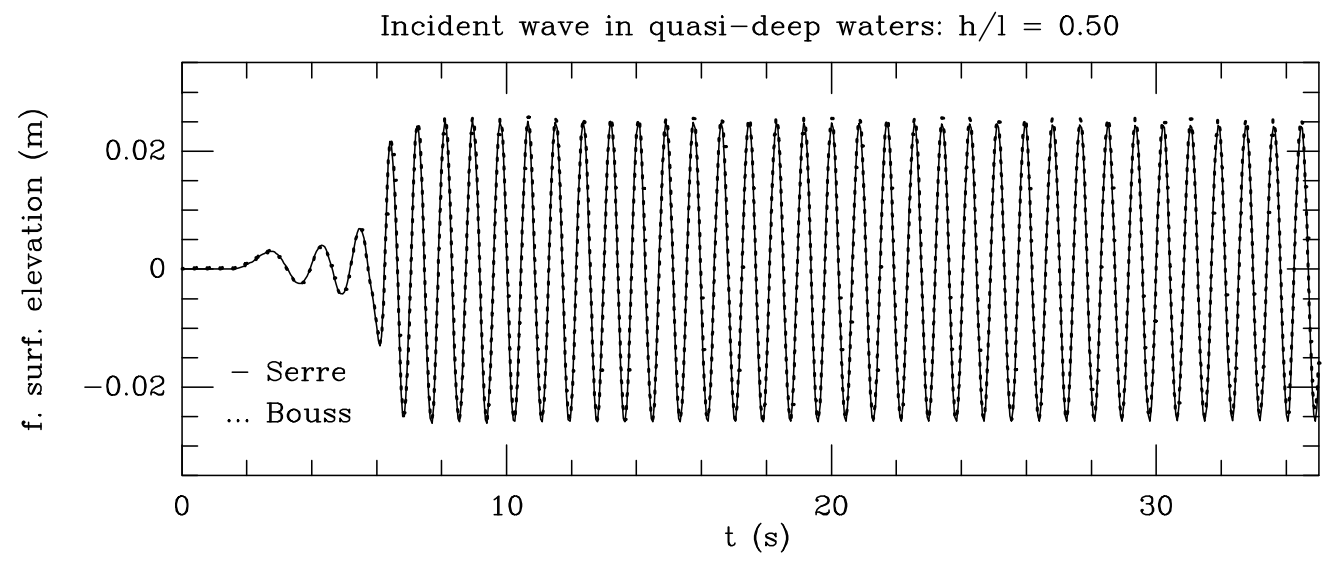

Fig. (4). Numerical results obtained with models (8) $(\cdots \cdots)$ ) and (9) $(-)$, in a depth gauge located at $x=5.0 \mathrm{~m}$ from the inlet section of the wave in the channel.

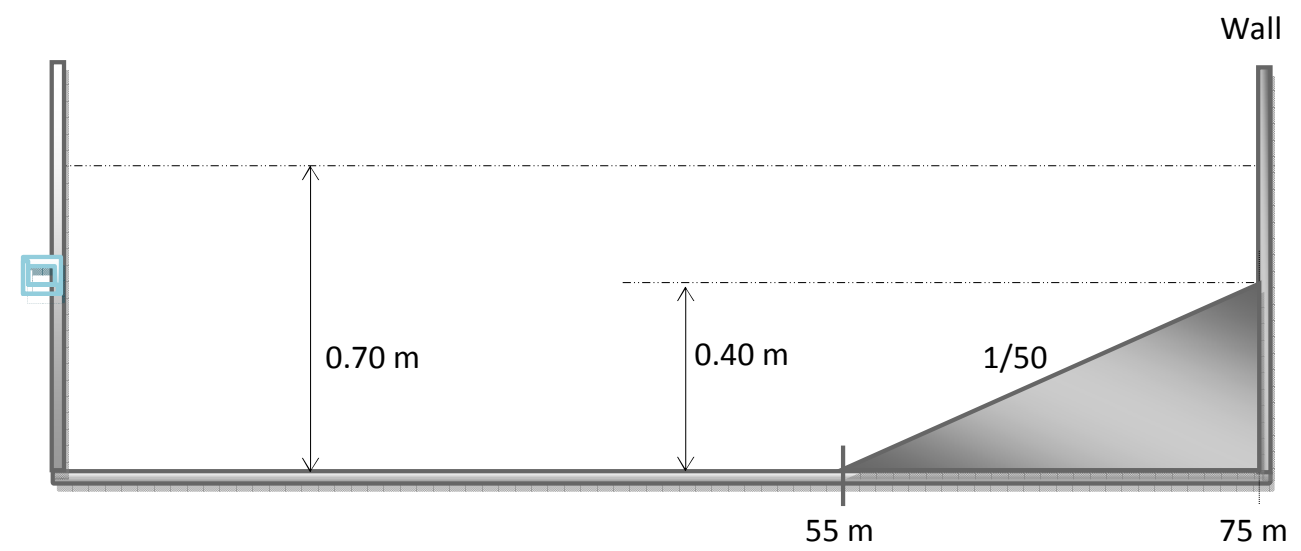

Fig. (5). Bathymetry for a solitary wave travelling up a slope and its reflection on a vertical wall (not in scale).

$0.12 \mathrm{~m}$ is initially centred at $x=25 \mathrm{~m}$. The computational domain was uniformly discretized with a spatial step $\Delta x=0.05 \mathrm{~m}$. A zero friction factor has been considered. Computations were carried out with a time step $\Delta t=0.010 \mathrm{~s}$. Fig. (6) compares numerical time series of surface elevation and test data at $x=72.75 \mathrm{~m}$.

Fig. (6) shows two peaks; the first one corresponding to the incident wave, and the second to the reflected wave. The extended Serre model predictions for both peaks are in conformity with the measurements. RMSE values equal to $0.0090 \mathrm{~m}$ and $0.0117 \mathrm{~m}$ were found in first and second peaks, respectively, for the wave height. Regarding the phase, there is a loss of approximately $0.05 \mathrm{~s}$ and of $0.10 \mathrm{~s}$ in those peaks.

Predictions of the extended Boussinesq equations for both peaks are less accurate. Particularly for the reflected peak which is overestimated in about $20 \%$. This result is not surprising, given the lower validity of the Boussinesq model for waves of higher relative amplitude. Indeed, this model assumes $O(\varepsilon)<<1$, contrary to the Serre model, which is $O(\varepsilon)=1$. A visual comparison of numerical results of the extended Boussinesq approximation (8) with a similar study performed by [19], using the extended Boussinesq model developed by [4], shows no relevant differences in the graphs.

\section{Periodic Wave Over an Underwater Bar}

Beji and Battjes [20] conducted experiments in a flume of $0.80 \mathrm{~m}$ wide with a submerged trapezoidal bar with slopes 1:10 (upstream) and 1:20 (downstream). Before and after the bar, the water depth is $0.40 \mathrm{~m}$, with a reduction to $0.10 \mathrm{~m}$ above the bar, as shown in Fig. (7). Experimental data obtained in this installation are available in the literature, and can be used for comparisons.

The measured data are compared with the numerical results of the extended Boussinesq model (8) and Serre equations (9), both improved with linear dispersive characteristics. Comparisons are made in three wave gauges located at $\mathrm{x}$ $=10.5 \mathrm{~m}, \mathrm{x}=13.5 \mathrm{~m}$ and $\mathrm{x}=17.3 \mathrm{~m}$. For this purpose, $\mathrm{a}$ regular incident wave case with height $0.02 \mathrm{~m}$, period $\mathrm{T}=2.02 \mathrm{~s}$ and wavelength $3.73 \mathrm{~m}$ has been simulated. The computational domain was discretized with a uniform grid interval $\Delta x=0.025 \mathrm{~m}$. A time step $\Delta t=0.0010 \mathrm{~s}$ was used. 


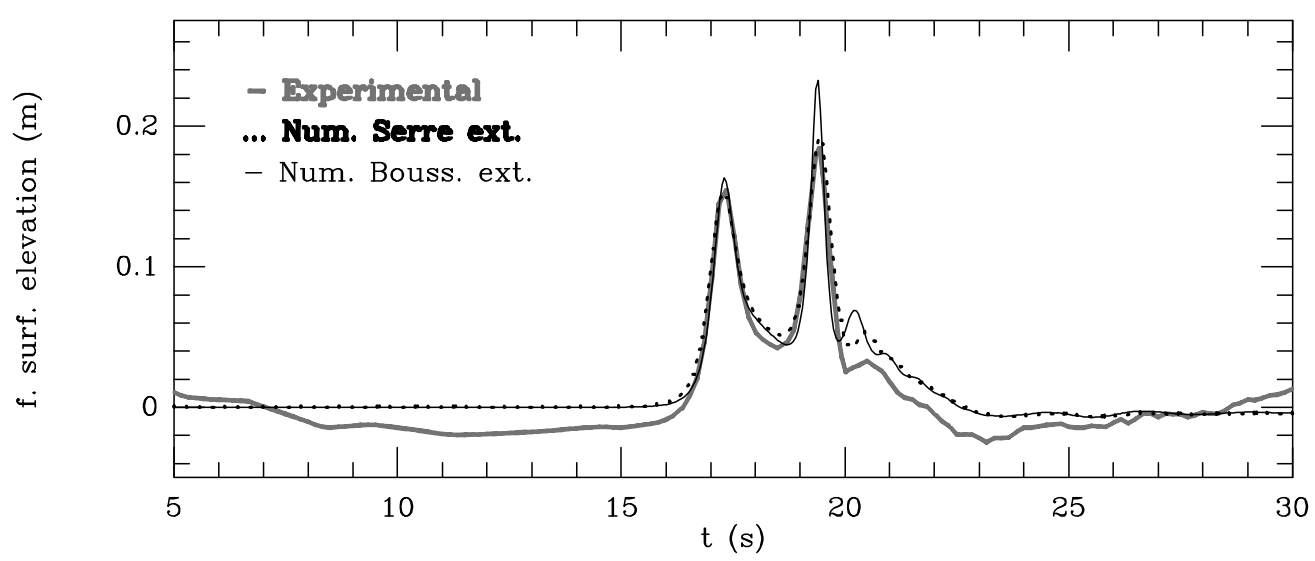

Fig. (6). Solitary wave travelling up a slope and its reflection on a vertical wall. Free surface elevation in a depth gauge located at $x=72.75$ m. Experimental ( $\square$ ); Serre extended ( $\cdots \cdots)$; Boussinesq extended $(-)$ ).

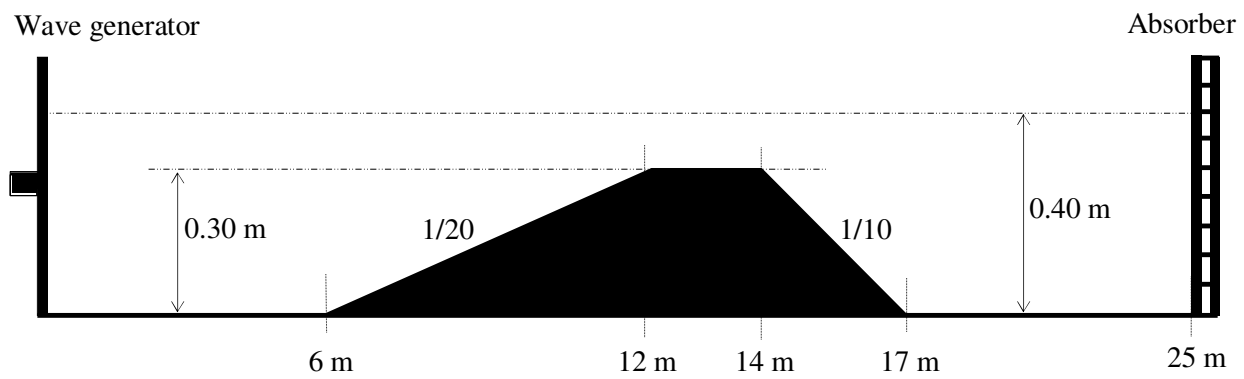

Fig. (7). Bathymetry for a periodic wave propagating over a bar (not in scale).

Both relations (14) and (15) were tested as input boundary conditions and the results, for this case, are quite close. Fig. (8) shows a comparison of test data with numerical results of both models (8) and (9).

As shown in the sequence of wave gauges, Fig. (8), as the wave shoals up the front face and over the bar, in very shallow water conditions, it steepens dramatically [Figs. (8a) and $(\mathbf{8 b})$ ] accumulating higher harmonics which are released on the downslope, producing an irregular pattern behind the bar, Fig. (8c). Globally, numerical results of the improved Serre and Boussinesq models agree quite well with the measured data. As expected, over the bar, in the gauge placed at $x=13.5 \mathrm{~m}$, the results of the extended Boussinesq model are generally slightly less accurate than those of the improved Serre model. $R M S E$ values equal to $0.0013 \mathrm{~m}$ and $0.0027 \mathrm{~m}$, for the wave height using Serre and Boussinesq approximations, respectively, were found in this gauge.

Also important is a comparison of the classical Serre model (6) with the extended Serre equations (7). The Serre model (6) is only valid for shallow waters, thus under conditions up to $h_{0} / l \leq 0.05$. In this experiment, the dispersion parameter $\left(\sigma=h_{0} / l\right)$ is greater than 0.05 (about 0.11 ) in front and behind the bar, and therefore affects the validity of the numerical outcomes. Due to the fact that over the bar there are very shallow water conditions $(\sigma \approx 0.03)$ the classical Serre equations are used considering the input boundary located at section $\mathrm{x}=13.5 \mathrm{~m}$, where the input signal is known (measured data). In this way, results of the Serre's classic model are not influenced, as would happen, by changes arising from the wave propagation before the bar, under intermediate water depths. Fig. (9) shows a comparison of numerical results of the classical Serre model (6) with the extended Serre equations (7), considering, in the first case, the input boundary at $\mathrm{x}=13.5 \mathrm{~m}$ (gauge signal).

The influence of additional terms of dispersive origin included in the extended Serre equations is clearly shown in Fig. (9). The classical Serre model results (dashed line) are clearly of lesser quality. It should be noted that this application also demonstrates the good behavior of our numerical model to propagate a complex signal imposed at boundary.

\section{CONCLUSIONS}

This paper presents a methodology to improve the linear dispersion characteristics of the classical Serre equations for variable depth, based on the procedure used by various researchers for Boussinesq type models. Extend the classical Serre equations for applications in intermediate water depths and quasi-deep waters is a significant contribution; in fact, these are the conditions usually found in nearshore zones.

The influence of the dispersion characteristics is clearly evidenced by the generation and propagation of waves in intermediate water depths, as is shown in cases of: (1) input and propagation of a periodic wave in a channel with $h_{0} / l=$ 0.50 , and (2) very demanding applications over bottoms with considerable slopes. The overall agreement of the extended 


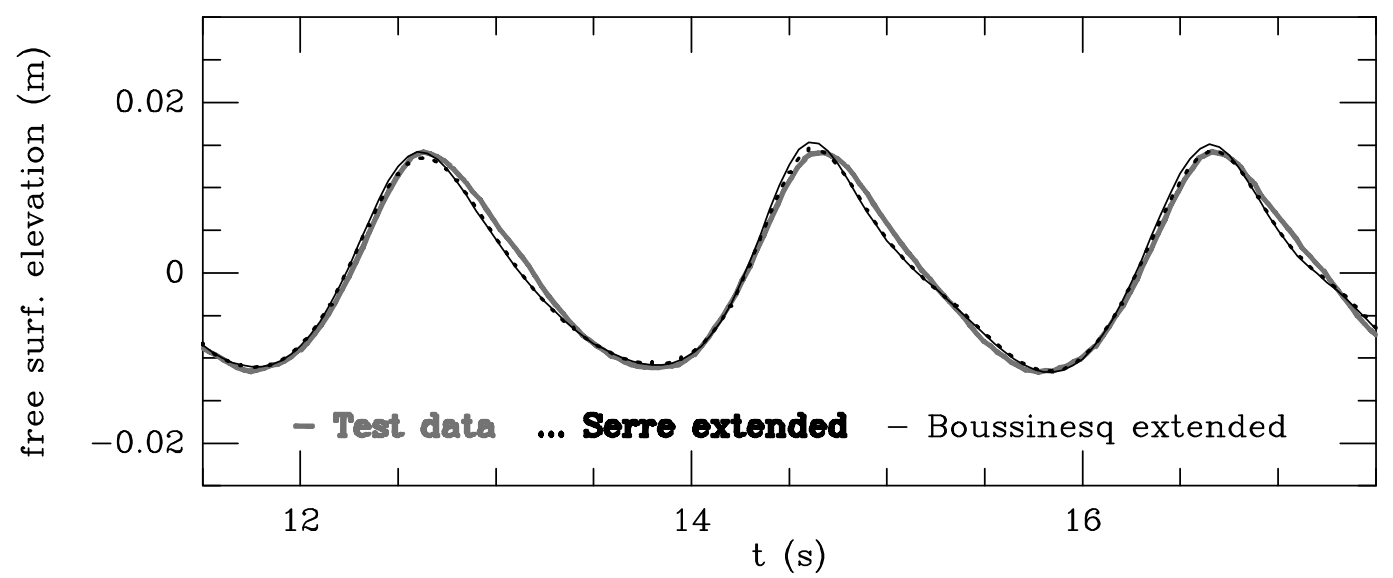

a)

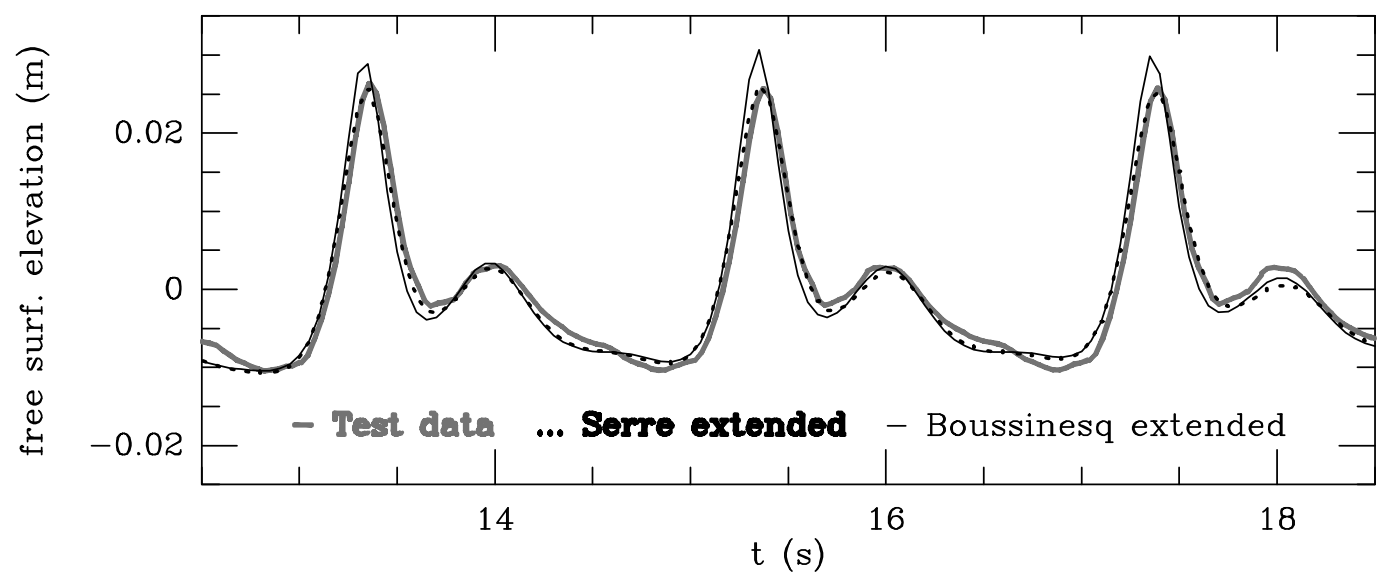

b)

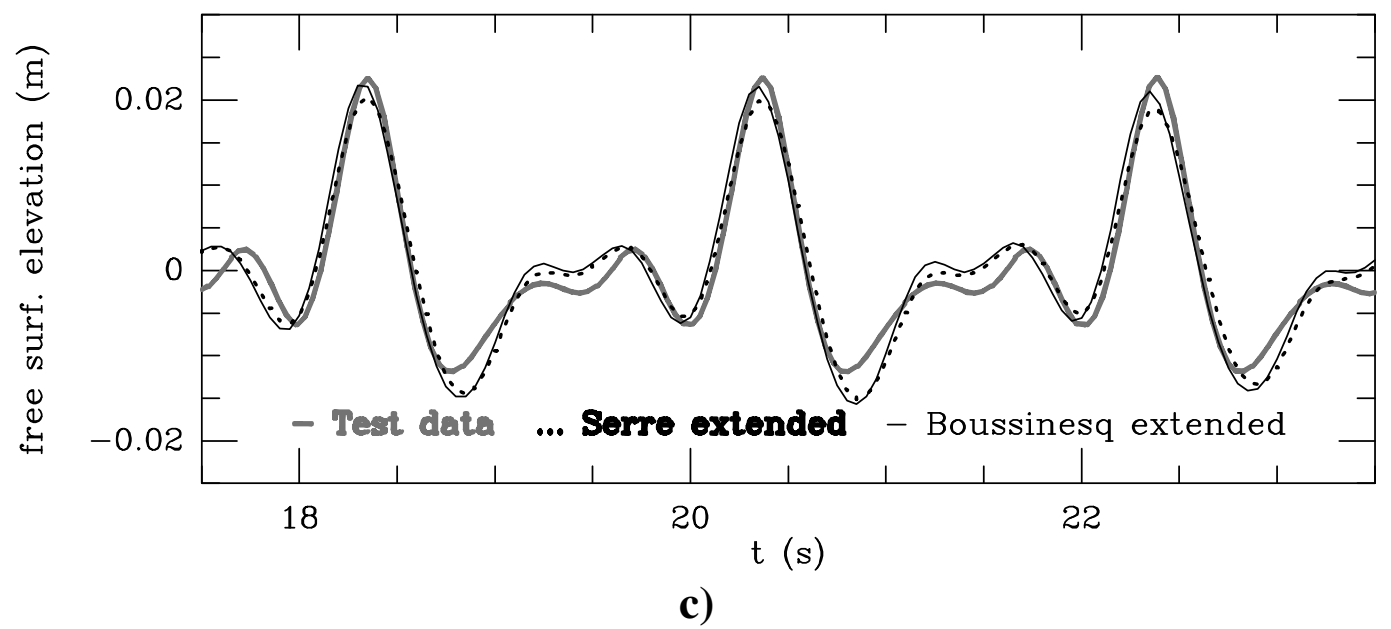

Fig. (8). Periodic wave propagating over a bar. Free surface evolution at three gauges installed at a) $x=10.5 \mathrm{~m}, \mathbf{b}) x=13.5 \mathrm{~m}$ and c) $x=17.3 \mathrm{~m}$. Experimental ( $\square$ ); Serre extended ( $\cdots \cdots)$; Boussinesq extended $(-)$. 


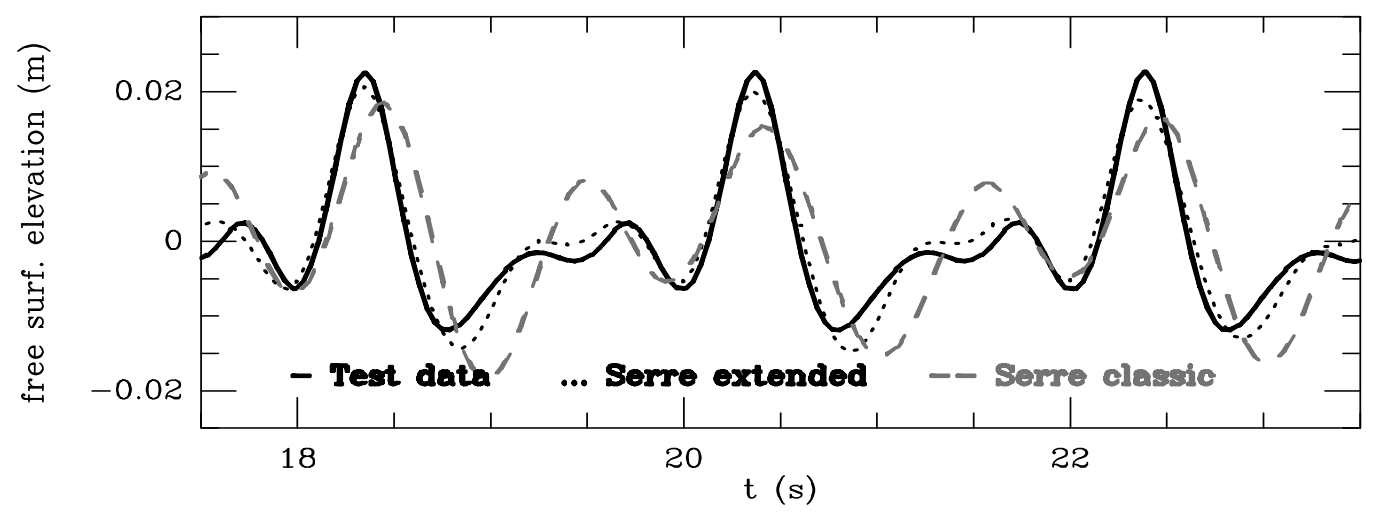

Fig. (9). Periodic wave propagating over a bar. Comparison of test data ( $(\cdots \cdots)$ and the classical Serre equations (6) (----).

) with numerical results of the extended Serre model (7)

Serre model with improved dispersion characteristics is very good both in shallow water conditions as in intermediate water depths and up to quasi-deep waters $\left(h_{0} / l \leq 0.50\right)$.

An extension to two dimensions in the horizontal plane is currently being developed and will be the subject of a second paper.

\section{CONFLICT OF INTEREST}

The authors confirm that this article content has no conflicts of interest.

\section{ACKNOWLEDGEMENTS}

Declared none.

\section{REFERENCES}

[1] P.A. Madsen, R. Murray, and O.R. Sørensen, "A New Form of the Boussineq Equations with Improved Linear Dispersion Characteristics”, Coastal Engineering, vol. 15, p. 371, 1991.

[2] P.A. Madsen, and O.R. Sørensen, "A New Form of the Boussineq Equations with Improved Linear Dispersion Characteristics: 2. A Slowly Varying Bathymetry", Coastal Engineering, vol. 18, p. 183; 1992.

[3] J. Boussinesq, "Théorie des ondes et des remous qui se propagent le long d'un canal rectangulaire horizontal", Journal of Mathématiques Pures et Appliquées, vol. 2, no. 17, pp. 55-108,1872.

[4] O. Nwogu, "Alternative form of Boussinesq equations for nearshore wave propagation", Journal of Waterway, Port, Coastal, and Ocean Engineering, vol. 119, pp. 618-638, 1993.

[5] G. Wei, and J.T. Kirby, "A time-dependent numerical code for extended Boussinesq equations", Journal of Waterway, Port, Coastal and Ocean Engineering, vol. 121, pp. 251-261, 1995.

[6] G. Wei, J.T. Kirby, S.T. Grilli, and R. Subramanya, "A fully nonlinear Boussinesq model for surface waves. I. Highly nonlinear, unsteady waves", Journal of Fluid Mechanics, vol. 294, pp. 71-92, 1995.

[7] M.F.G. Gobbi, J.T. Kirby, and G. Wei, "A fully nonlinear Boussinesq model for surface waves. Part 2. Extension to $O(k h)^{4,}$, Journal of Fluid Mechanics, vol. 405, pp. 181-210, 2000.

[8] P. Lynett, and P. L.-F. Liu, "Modeling Wave Generation, Evolution, and Interaction with Depth Integrated, Dispersive Wave Equa- tions COULWAVE Code Manual', Cornell University Long and Intermediate Wave Modeling Package, Royal Socity: UK, 2002.

[9] S. Beji, and K. Nadaoka, "Formal derivation and numerical modelling of the improved Boussinesq equations for varying depth", Ocean Engineering, vol. 23; p. 691, 1996.

[10] Z.B. Liu, and Z.C. Sun, "Two sets of higher-order Boussinesq-type equations for water waves", Ocean Engineering, vol. 32, pp. 1296$1310,2005$.

[11] F. Serre, "Contribution à l'étude des écoulements permanents et variables dans les canaux", La Houille Blanche, vol. 8, pp. 374$388 \& 830-872,1953$.

[12] A.E. Green, and P.M. Naghdi, "A derivation of equations for wave propagation in water of variable depth", Journal of Fluid Mecanics, vol. 78 (2), pp. 237-246, 1976.

[13] J.S. Antunes do Carmo, "Wave-current interactions over bottom with appreciable variations both in space and time", Journal of $A d$ vances in Engineering Software, vol. 41, no. 2, pp. 295-305, 2010.

[14] R. Cienfuegos, E. Barthélemy, and P. Bonneton, "A fourth-order compact finite volume scheme for fully nonlinear and weakly dispersive Boussinesq-type equations. Part I: model development and analysis", International Journal for Numerical Methods in Fluids, vol. 51, pp. 1217-1253, 2006.

[15] R.F. Carvalho, and J.S. Antunes do Carmo, "Numerical and experimental modelling of the generation and propagation of waves caused by landslides into reservoirs and their effects on dams", Proceedings of RIVER FLOW 2006 IAHR Conference, Lisbon (LNEC), 6-8 September, 2006. In: Taylor \& Francis: UK, vol.1, pp. 483-492, 2006.

[16] J.S. Antunes do Carmo, "Boussinesq-type equations with improved dispersion characteristics. Applications", Journal of Water Resources vol. 32, no. 2, pp. 07-16, 2011 (in Portuguese).

[17] D.J. Korteweg, and G. De Vries, "On the change of form of long waves advancing in a rectangular canal, and on a new type of stationary waves", Philosophical Magazine, vol. 39/5, pp. 422-443, 1895.

[18] N. Dodd, "A numerical model of wave run-up, overtopping and regeneration", ASCE Journal of Waterway, Port, Coastal and Ocean Engineering, vol. 124, no. 2, pp. 73-80, 1998.

[19] M. Walkley, and M. Berzins, "A finite element method for the onedimensional extended Boussinesq equations", International Journal for Numerical Methods In Fluids, vol. 29, pp. 143-157, 1999.

[20] S. Beji, and J.A. Battjes, "Experimental investigations of wave propagation over a bar", Coastal Engeenring., vol. 19, no. (1,2), pp. 151-162, 1993.

Received: April 16, 2013

Revised: June 23, 2013

Accepted: June 25, 2013

C José Simão Antunes do Carmo; Licensee Bentham Open.

This is an open access article licensed under the terms of the Creative Commons Attribution Non-Commercial License (http://creativecommons.org/licenses/by-nc/3.0/) which permits unrestricted, non-commercial use, distribution and reproduction in any medium, provided the work is properly cited. 